\title{
Metformin treatment confers protection of the optic nerve following photoreceptor degeneration
}

\author{
Sohair A. Eltony, Heba S. Mohaseb, Manal M. Sayed, Amel A. Ahmed \\ Department of Histology and Cell Biology, Faculty of Medicine, Assiut University, Assiut, Egypt
}

\begin{abstract}
Acquired or inherited or photoreceptor loss causes retinal ganglion cell loss and ultimately axonal transport alteration. Thus, therapies should be applied early during photoreceptors degeneration before the remodeling process reaches the inner retina. This study aimed to evaluate the protective effect of metformin on the rat optic nerve following photoreceptors loss induced by N-Ethyl- $N$-nitrosourea (ENU). Eighteen adults male Wistar rats were divided into two groups. Group I: normal vehicle control $(n=6)$. Group II: ENU-induced photoreceptors degeneration $(n=12)$ received a single intraperitoneal injection of ENU at a dose of $600 \mathrm{mg} / \mathrm{kg}$. Rats in group II were equally divided into two subgroups: IIa: photoreceptor degeneration induced group and IIb: metformin treated group ( $200 \mathrm{mg} / \mathrm{kg})$ for 7 days. Specimens from the optic nerve were processed for light and electron microscopy. In ENU treated group, the optic nerve revealed reduction in the diameter of the optic nerve fibers and thinning of myelin sheath with morphological changes in the glia (astrocytes, oligodendrocytes, and microglia). Caspase-3 (apoptotic marker), iNOS (oxidative stress marker) and CD68 (macrophage marker) expression increased. In metformin-treated group, the diameter of optic nerve fibers and myelin sheath thickness increased with improvement of the deterioration in the glia. Caspase-3, iNOS and CD68 expression decreased. Metformin ameliorates the histological changes of the rat optic nerve following photoreceptors loss induced by ENU.
\end{abstract}

Key words: Optic nerve, Retinal degeneration, Metformin, Oxidative stress, Astrocytes

Received December 21, 2020; Revised January 28, 2021; Accepted January 29, 2021

\section{Introduction}

Acquired or inherited photoreceptors loss initiates a chain of events, known as "retinal remodeling", that ends in retinal ganglion cell (RGC) death [1]. As RGCs are the most relevant neurons in the inner retina as their axons form the optic nerve that sends the visual information to the visual cortex. Thus, therapies should be applied early during photorecep-

\section{Corresponding author:}

Sohair A. Eltony (iD)

Department of Histology and Cell Biology, Faculty of Medicine, Assiut University, Assiut 71515, Egypt

E-mail: sohair_eltony@yahoo.com, sohair@aun.edu.eg tors degeneration before the remodeling process reaches the inner retina [1].

$\mathrm{N}$-Ethyl- $\mathrm{N}$-nitrosourea (ENU) is a DNA alkylating agent that targets the retinal photoreceptor cells. It has been used to create photoreceptor degenerative diseases animal model via apoptosis [2], such as age-related macular degeneration and retinitis pigmentosa $(\mathrm{RP})[3,4]$.

Metformin is a well-tested drug used to treat type 2 diabetes, and recently it has been reported to have beneficial effects in neurodegenerative diseases [5]. It was demonstrated that metformin ameliorates the neuropathic effects of acrylamide in rats by decreasing caspase 3 activity and up regulating bcl2 expression [6].

So, this study was carried out to evaluate the protective effect of metformin on the rat optic nerve following photore-

\section{Copyright ( 2021 . Anatomy \& Cell Biology}

This is an Open Access article distributed under the terms of the Creative Commons Attribution Non-Commercial License (http://creativecommons.org/licenses/by-nc/4.0/) which permits unrestricted non-commercial use, distribution, and reproduction in any medium, provided the original work is properly cited. 
ceptors loss induced by ENU using light and electron microscope.

\section{Materials and Methods}

A total number of 18 adult 5 months old male Wistar rats (200 g body weight) were used in this study. They were purchased from the Central Animal House, Faculty of Medicine, Assiut University. All animal procedures were in accordance with the standards set forth in the guidelines for the care and use of experimental animals by the Committee for the Purpose of Supervision of Experiments on Animals and according to the National Institute of Health protocol and approved by the Institutional Ethics Committee of Assiut University.

\section{Animal groups: The animals were divided into 2 groups}

Group (I): (normal vehicle control) consisted of 6 rats that received a single intraperitoneal injection of $0.5 \mathrm{ml}$ physiologic saline. Group (II): (ENU-induced photoreceptor degeneration) consisted of 12 rats that received a single intraperitoneal injection of ENU (purchased from Sigma Chemical Co., St. Louis, Mo, USA) at a dose of $600 \mathrm{mg} / \mathrm{kg}$ freshly dissolved in $0.5 \mathrm{ml}$ physiologic saline [7]. Rats in group II were equally divided into two subgroups: Group (IIa) (photoreceptor degeneration group) consisted of 6 rats that received a daily intraperitoneal injection of $0.5 \mathrm{ml}$ physiologic saline, for 7 days starting one day before ENU administration. Group (IIb) (metformin-treated group) consisted of 6 rats that received metformin (purchased from Nasr Company for Pharmaceutical Chemicals) by intraperitoneal injection once daily at a dose of $200 \mathrm{mg} / \mathrm{kg}$ dissolved in $0.5 \mathrm{ml}$ physiologic saline, for 7 days. Metformin injection was started one day before ENU administration $[8,9]$.

The animals were anaesthetized with ether, their hearts were exposed, and then perfusion was done.

For a light microscope, three rats from each animal group were used and perfused intracardially with a $10 \%$ formaldehyde solution. The retina was separated, and the optic nerve (intraorbital part) was transected 1 to $2 \mathrm{~mm}$ behind the globe. Paraffin sections $(5 \mu \mathrm{m})$ were cut using a microtome (Leica RM 2125RT, Leica microsystems, Wetzlar, Germany) and every 10th section was stained with hematoxylin-eosin [10]. Some sections were stained with Mallory's phosphotungstic acid hematoxylin (PTAH) for demonstration of glial fibrils within astrocytes in the optic nerve [10].
For electron microscope, three rats from each group were perfused intracardially with $4 \%$ glutaraldehyde in cacodylate buffer (pH 7.4). Specimens from the retina and optic nerve were cut into thin slices $(1 \times 1 \mathrm{~mm})$ and immersed in the fixative for 24 hours and post fixed in 1\% osmium tetroxide in phosphate buffer for two hours [11]. Semi-thin sections $(0.5-1 \mu \mathrm{m})$ were cut on the ultramicrotome (LKB Bromma 8800 UltratomeR III, 3518, Sweden) and stained with $1 \%$ toluidine blue ( $\mathrm{pH}$ 7.3) for examination on a light microscope (Bx50. Model Bx50F-3, SC09160; Olympus, Tokyo, Japan). Ultrathin sections $(50-80 \mathrm{~nm})$ were cut from selected areas of the blocks on a Reichert ultramicrotome (Leica WILDM3Z, 89386, Wien, Austria) and contrasted with uranyl acetate and lead citrate. These sections were examined using the transmission electron microscope (Jeol E.M.-100 CX11; Japanese electron optic laboratory, Tokyo, Japan). All processes were done in Electron microscopic unit at Assiut University.

\section{Immunohistochemical study}

Expression of Caspase-3 (apoptotic marker), CD68 (macrophage marker), and inducible nitric oxide synthase (iNOS) (oxidative stress marker) was detected in formalin-fixed paraffin-embedded sections. The Caspase-3 (CPP32) Ab-4, Rabbit Polyclonal Antibody (Thermo Fisher Scientific, Fremont, CA 94538-7310, USA), the (iNOS) Rabbit Polyclonal Antibody (Thermo Fisher Scientific, Fremont, CA 94538 6406, USA), and the CD68 (Monoclonal mouse antibody) Ab-3, (Clone KP1 Lab Vision Co., Neo Markers Inc/Lab Vision, Fremont, CA, USA) were used at 1:100 dilution for 30 minutes at room temperature.

Sections were processed according to the manufacture instructions using the universal kit (Ecno Tek HRP AntiPolyvalent, DAB) (ScyTek Laboratories, Inc., 205 South 600 West Logan, UT 84321 USA). After completion of the reaction, counterstaining was done using Mayer's hematoxylin, dehydrated and cover-slipped using DPX (Oxford laboratory reagents, Bombay, India).

\section{Morphometric study}

Using the Image J software program, the total retinal thickness (from the internal limiting membrane to the pigment epithelium) was measured in 5 micrographs of toluidine blue stained semithin sections ( $\times 400$ magnification) from three different animals for each group. The number of astrocytes per $\mathrm{mm}^{2}$ was counted in optic nerve in 5 micrographs of PTAH-stained paraffin sections $(\times 400$ magnifica- 

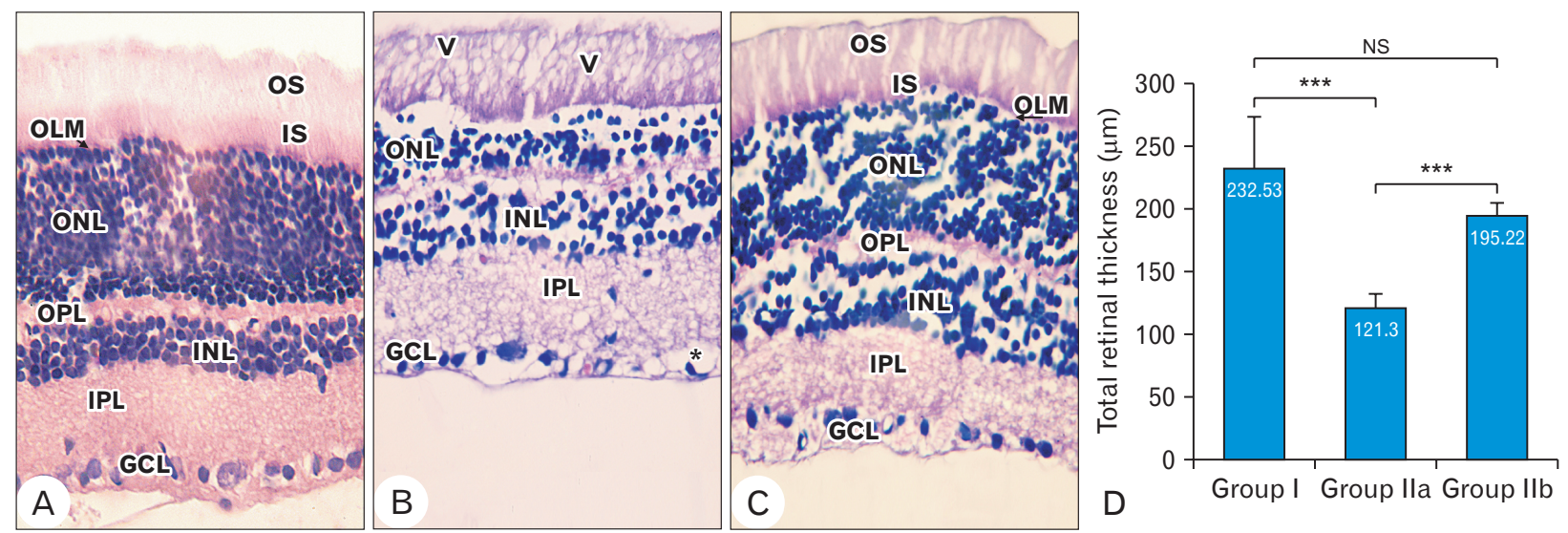

Fig. 1. Photomicrographs, paraffin sections stained with H\&E, $\times 1,000$. OSs and ISs of photoreceptors, OLM, ONL, OPL, INL, IPL, and GCL. (A) Group I showing well defined retinal layers. (B) Group IIa showing V, less populated and widely separated photoreceptor nuclei in the ONL, empty spaces $\left(^{*}\right)$ in the GCL. (C) Group IIb showing decreased photoreceptor degenerative changes compared to group IIa. (D) Comparison of the total retinal thickness $(\mu \mathrm{m})$ in the studied groups. GCL, ganglion cell layer; INL, inner nuclear layer; IPL, inner plexiform layer; IS, inner segment; NS, no significant; OLM, outer limiting membrane; ONL, outer nuclear layer; OPL, outer plexiform layer; OS, outer segment; V, vacuoles. Data are expressed as mean \pm standard deviation. ${ }^{* * *} P<0.000$. No significant difference, $P<0.08$.

tion) from three different animals for each group. The number of Caspase-3, iNOS and CD68 positive cells per $\mathrm{mm}^{2}$ was counted in optic nerve in 5 micrographs of immunostained paraffin sections ( $\times 400$ magnification) from three different animals for each group. The diameter of optic nerve fibers $(\mu \mathrm{m})$ and thickness of the myelin sheath of optic nerve fibers $(\mu \mathrm{m})$ were measures in 5 micrographs of ultrathin sections $(\times 3,600$ magnification) from three different animals for each group.

\section{Statistical analysis}

The morphometric data of each animal group were statistically analyzed using the computer statistics Prism-5.0 package (GraphPad Software, Inc., San Diego, CA, USA). One-way analysis of variance (ANOVA) followed by Turkey's test as a post-test was employed to compare the studied animal group. The results were expressed as mean \pm standard deviation (SD). A $P$-value $<0.05$ was considered significant.

\section{Results}

\section{Light microscopy}

\section{Histological results}

In group I, the retinal layers were well defined (Fig. 1A). The mean total retinal thickness was $232.53 \pm 40.81 \mu \mathrm{m}$, ranged from 200.8 to $286.0 \mu \mathrm{m}$ (Fig. 1D). The optic nerve was surrounded by the pia mater and divided into fascicles by thin connective tissue septa (Fig. 2A). It is formed of
RGCs axons and glial cells. The axons appeared myelinated with variable sizes (Fig. 2D). Oligodendrocytes had small dark nuclei while astrocytes were characterized by large lightly stained nuclei (Fig. 2D). Astrocytes and their long processes appeared dark blue in color with PTAH stain (Fig. $2 \mathrm{G})$. Their mean number per $\mathrm{mm}^{2}$ was $16 \pm 2.943$, ranged from 13 to 20 (Fig. 2J).

In group IIa, ENU treated retina revealed photoreceptor degenerative changes in the form of vacuolations and loss of photoreceptor nuclei. Also, some ganglion cells were lost leaving empty spaces (Fig. 1B). The mean total retinal thickness was significantly decreased $(P$-value $<0.000)$ compared to group I. It was $121.30 \pm 11.10 \mu \mathrm{m}$, ranged from 106.4 to 131.3 $\mu \mathrm{m}$ (Fig. 1D). The optic nerve showed less organized connective tissue septa (Fig. 2B). There was a reduction in the myelinated nerve axons compared to group I (Fig. 2E). Using PTAH stain, the optic nerve showed astrocytes with negatively stained processes (Fig. $2 \mathrm{H}$ ). Their mean number per $\mathrm{mm}^{2}$ was significantly decreased $(P$-value $<0.0003)$ compared to group I. It was $5.75 \pm 1.707$, ranged from 4 to 8 (Fig. 2 J).

In group IIb, metformin treated retina revealed decreased evidence of photoreceptor degenerative changes induced by ENU (Fig. 1C). The mean total retinal thickness was significantly increased $(P$-value $<0.000)$ compared to group IIa. It was 195.22 \pm 9.19 , ranged from 186.4-208.8 (Fig. 1D). The optic nerve connective tissue septa were still less organized compared to group I (Fig. 2C). The myelinated nerve axons were increased compared to group IIa (Fig. 2F). Astrocytic 

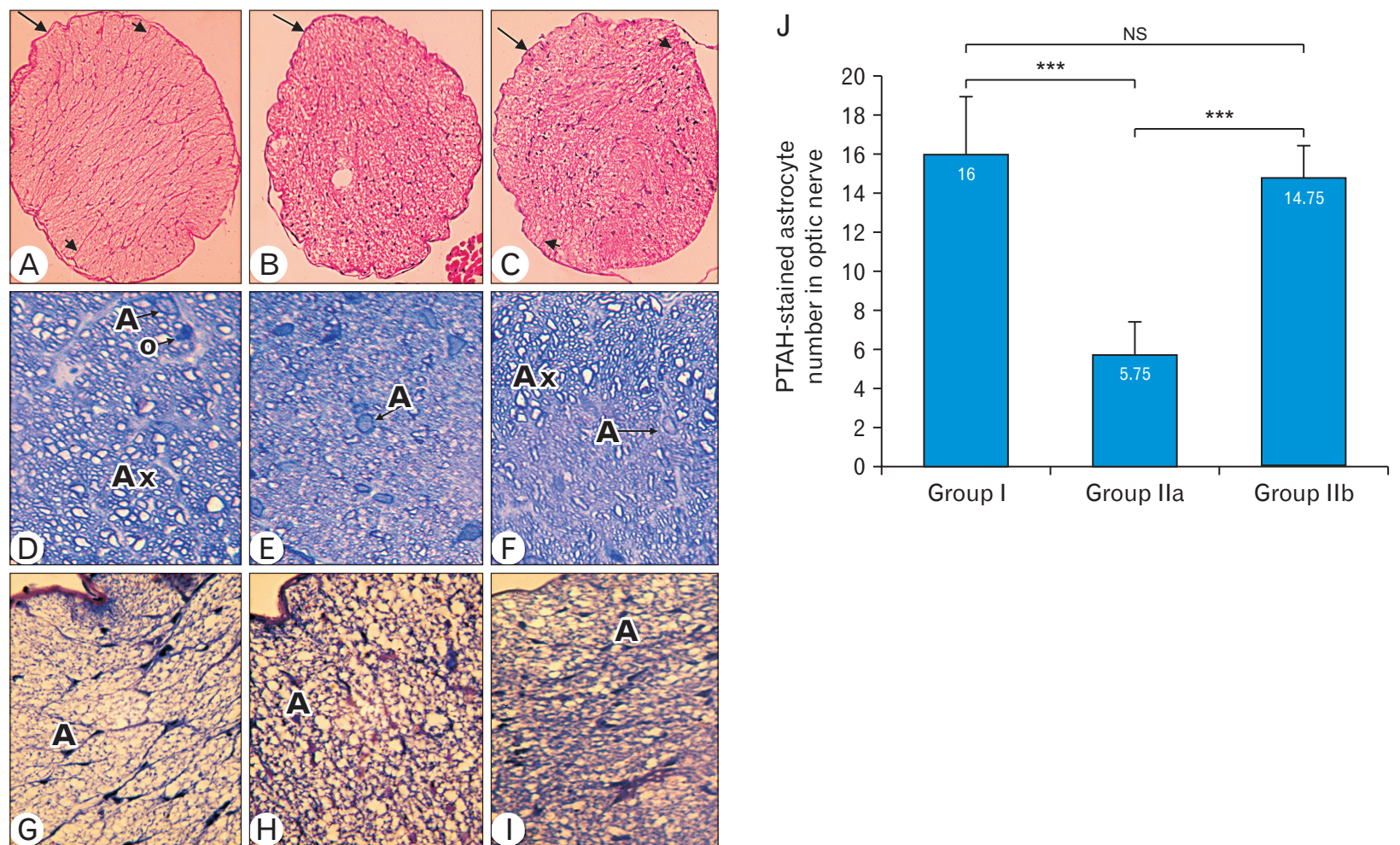

Fig. 2. Photomicrographs, group I (A, D, G), group IIa (B, E, H), group IIb (C, F, I). Paraffin sections stained by H\&E, $\times 1,000$ (A-C). Semithin sections stained with toluidine blue, $\times 1,000$ (D-F). Paraffin sections stained with PTAH, $\times 1,000$ (G-I). (A) Group I showing the optic nerve surrounded by the pia matter (arrow). Thin connective tissue septa (arrow head) extended into the nerve dividing it into fascicles. (B) Group IIa showing the optic nerve with less organized septa. Pia matter (arrow). (C) Group IIb showing that the septa (arrow head) are still less organized. Pia matter (arrow). (D) Group I showing myelinated Ax, A, O. (E) Group IIa showing reduction in the myelinated Ax compared to group I. A. (F) Group IIb showing myelinated Ax, A. (G) Group I showing A and their long processes. (H) Group IIb showing A with negatively stained processes. (I) Group IIb showing A with positively stained processes. (J) Comparison of the PTAH-stained A number in optic nerve of the studied groups. A, astrocyte; Ax, axons; NS, no significant; O, oligodendrocyte; PTAH, phosphotungstic acid hematoxylin. Data are expressed as mean \pm standard deviation. ${ }^{* * *} P<0.000$. No significant difference, $P<0.71$.
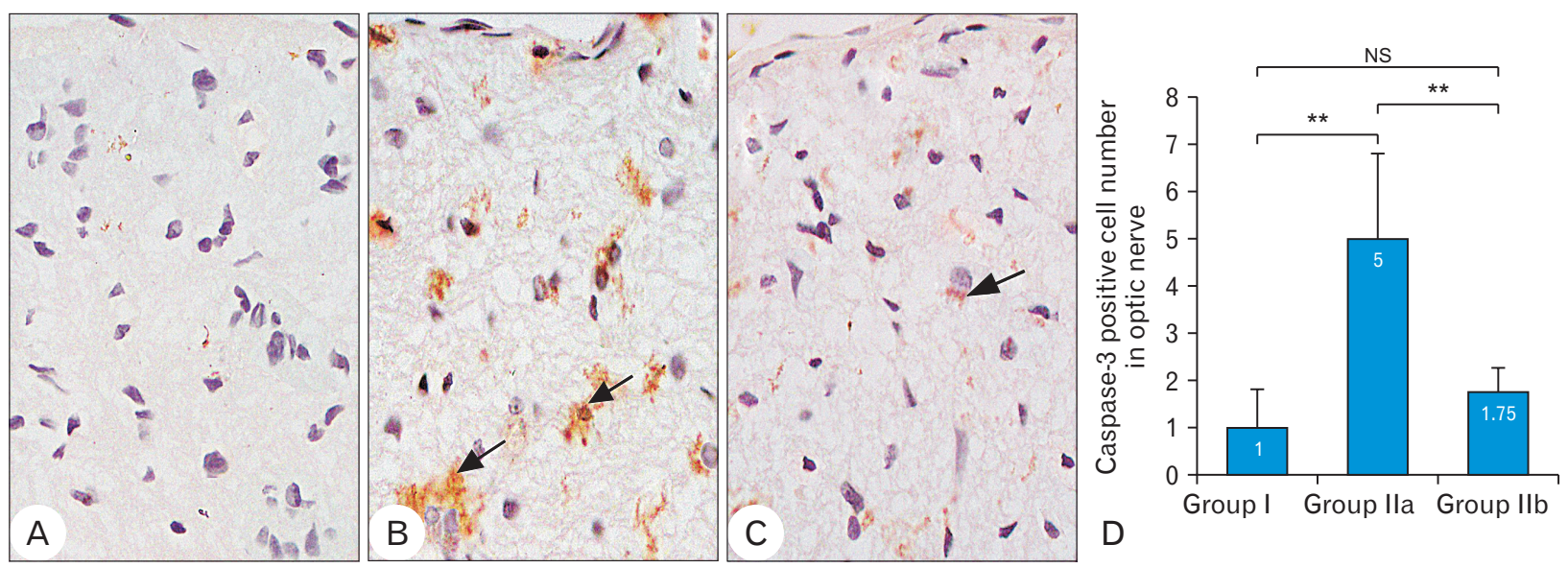

Fig. 3. Photomicrographs, caspase-3 immunostained paraffin sections, $\times 1,000$. (A) Group I showing mild positive caspase-3 reaction. (B) Group IIa showing strong positive caspase-3 reaction (arrow). (C) Group IIb showing decreased caspase-3 immunoreactivity compared to group IIa. (D) Comparison of caspase-3 positive cell number in optic nerve of the studied groups. NS, no significant. Data are expressed as mean \pm standard deviation. ${ }^{* *} P<0.01$. No significant difference, $P<0.659$. 
cell bodies and processes appeared dark blue in color with PTAH (Fig. 2I). Their mean number per $\mathrm{mm}^{2}$ was significantly increased $(P$-value $<0.0007)$ compared to group IIa. It was $14.75 \pm 1.707$, ranged from 13 to 17 (Fig. 2J).

\section{Immunohistochemical results}

Group I optic nerve showed mild positive caspase-3 reaction (Fig. 3A). The mean caspase positive cell number per $\mathrm{mm}^{2}$ was $1 \pm 0.8165$, ranged from 0 to 2 (Fig. 3D). While in group IIa, caspase-3 expression was strong positive in glial cells (Fig. 3B). The mean caspase positive cell number per $\mathrm{mm}^{2}$ was significantly increased $(P$-value $<0.0027)$ compared to group I. It was $5 \pm 1.826$, ranged from 3 to 7 (Fig. 3D). The optic nerve of group IIb showed decreased caspase- 3 immunoreactivity compared to group IIa (Fig. 3C). The mean caspase positive cell number per $\mathrm{mm}^{2}$ was significantly decreased $(P$ value $<0.0097)$ compared to group IIa. It was $1.75 \pm 0.5$, ranged from 1 to 2 (Fig. 3D).

The expression of iNOS immunoreactivity was mildly positive in group I optic nerve (Fig. 4A). The mean iNOS positive cell number per $\mathrm{mm}^{2}$ was $7 \pm 1.0$, ranged from 6 to 8 (Fig. 4D). While group IIa showed strong positive iNOS reaction in glial cells (Fig. 4B). The mean number of iNOS positive cells per $\mathrm{mm}^{2}$ was significantly increased $(P$-value $<0.0004)$ compared
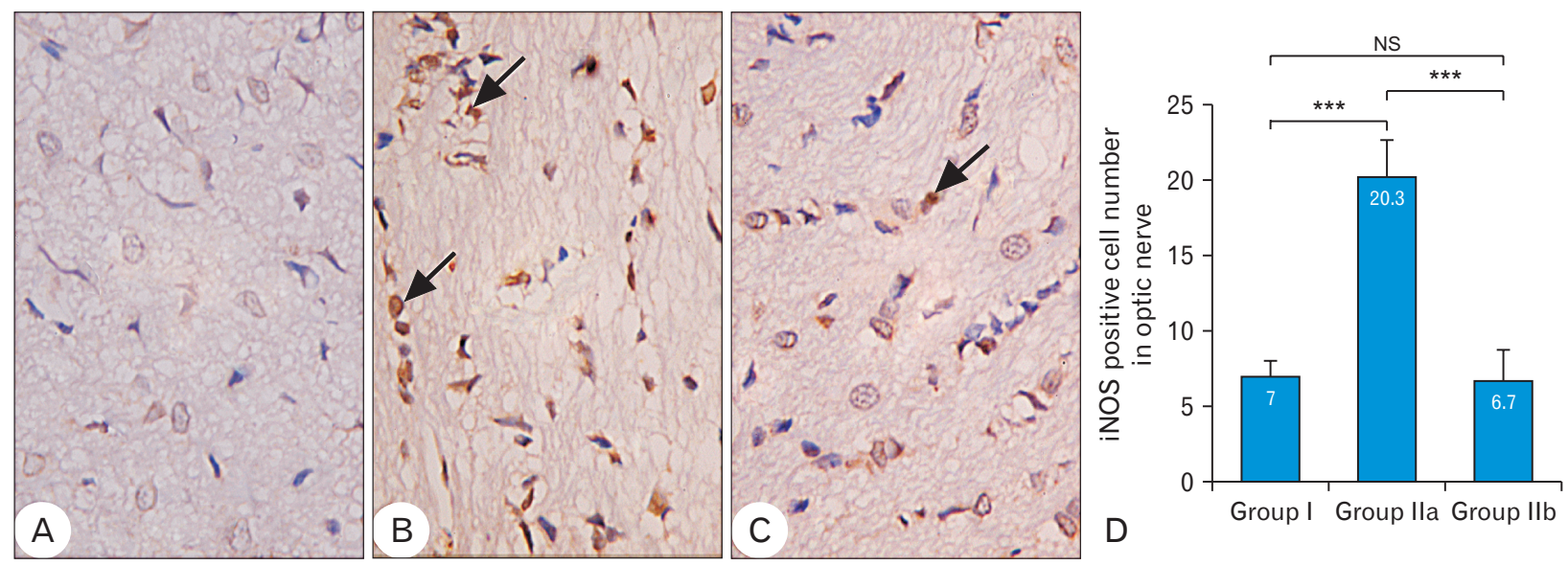

Fig. 4. Photomicrographs, iNOS immunostained paraffin sections, $\times 1,000$. (A) Group I showing mild positive iNOS reaction. (B) Group IIa showing strong positive iNOS reaction (arrow). (C) Group IIb showing decreased iNOS immunoreactivity (arrow) compared to group IIa. (D) Comparison of iNOS positive cell number in optic nerve of the studied groups. NS, no significant. Data are expressed as mean \pm standard deviation. ${ }^{* * *} P<0.001$. No significant difference, $P<0.9767$.
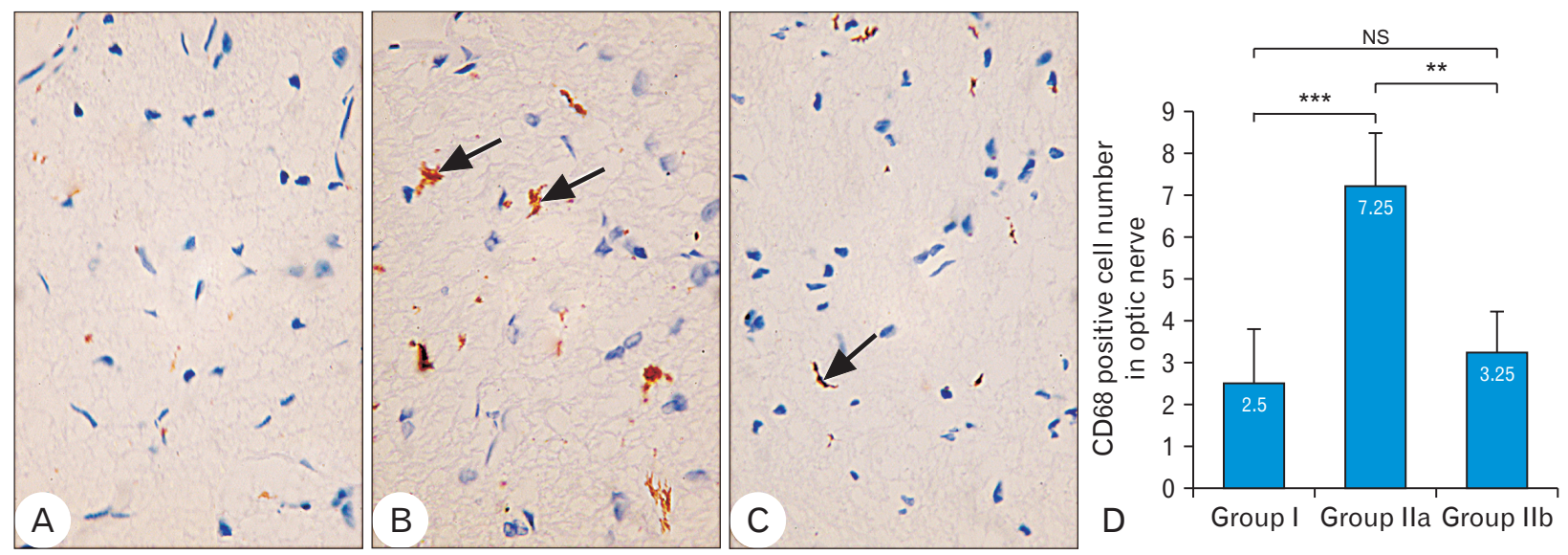

Fig. 5. Photomicrographs, CD68 immunostained paraffin sections, $\times 1,000$. (A) Group I showing mild positive CD68 reaction. (B) Group IIa showing strong positive CD68 reaction (arrow). (C) Group IIb showing decreased CD68 immunoreactivity (arrow) compared to group IIa. (D) Comparison of the CD68 positive cell number in optic nerve of the studied groups. NS, no significant. Data are expressed as mean \pm standard deviation. ${ }^{* *} P<0.001$. ${ }^{* *} P<0.01$. No significant difference, $P<0.6538$. 
to group I. It was $20.3 \pm 2.5166$, ranged from 18 to 23 (Fig. 4D). Group IIb optic nerve showed decreased iNOS immunoreactivity compared to group IIa (Fig. 4C). The mean number of iNOS positive cells per $\mathrm{mm}^{2}$ was significantly decreased $(P$-value $<0.0004)$ compared to group IIa. It was $6.7 \pm 2.0816$, ranged from 5 to 9 (Fig. 4D).

CD68 immunoreactivity was mild positive in group I optic nerve (Fig. 5A). The mean CD68 positive cell number per $\mathrm{mm}^{2}$ was $2.5 \pm 1.290994$, ranged from 1 to 4 (Fig. 5D). While in group IIa, the optic nerve showed strong positive CD68 reaction in glial cells (Fig. 5B). The mean number of CD68 positive cells per $\mathrm{mm}^{2}$ was significantly increased $(P$ value $<0.0008)$ compared to group I. It was $7.25 \pm 1.258306$, ranged from 6 to 9 (Fig. 5D). The optic nerve of group IIb showed decreased CD68 immunoreactivity compared to group IIa (Fig. 5C). The mean number of CD68 positive cells per $\mathrm{mm}^{2}$ was significantly decreased $(P$-value $<0.0025)$ compared to group IIa. It was $3.25 \pm 0.957427$, ranged from 2 to 4 (Fig. 5D).

\section{Electron microscopy}

In group I, the myelinated optic nerve fibers were arranged in fascicles surrounded by astrocytic processes (Fig. 6A). The mean diameter of optic nerve fibers was $4.825 \pm 0.81802$, ranged from 3.8 to 5.7 (Fig. 7A). The mean thickness of myelin sheath of optic nerve fibers was $0.605 \pm 0.099$, ranged from 0.53 to 0.75 (Fig. 7B). Astrocytes had large euchromatic nucleus with thin peripheral heterochromatin and long processes containing bundles of filaments (Fig. 6A). Oligodendrocytes had ovoid nucleus with peripheral clumped chromatin and moderate electron-dense cytoplasm (Fig. 6A). Microglia had small oval heterochro-

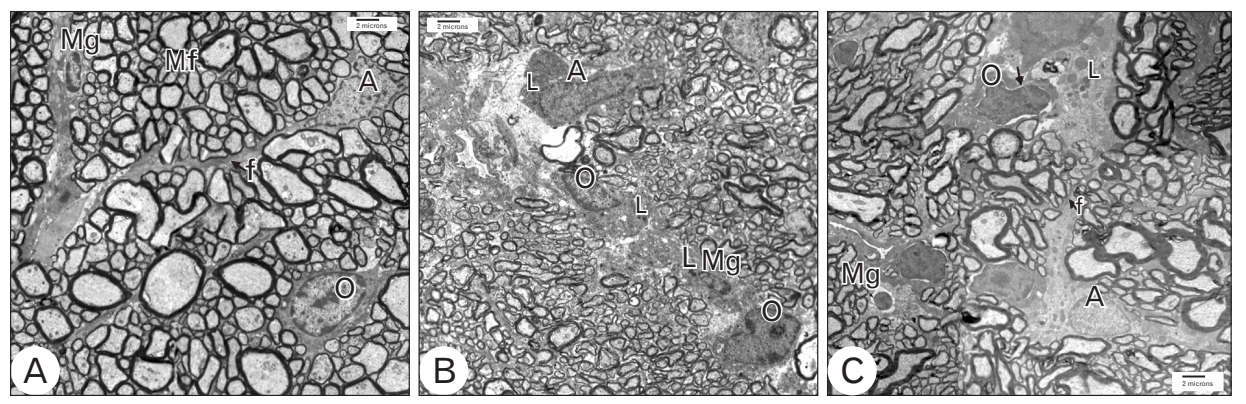

Fig. 6. Electron micrographs, Scale bar $=2 \mu \mathrm{m}, \times 3,600$. (A) Group I optic nerve shows A with large euchromatic nucleus and long processes containing bundles of $\mathrm{f}$. $\mathrm{O}$ with ovoid nucleus and peripheral clumped chromatin. Mg with small oval heterochromatic nucleus. (B) Group IIa optic nerve shows A with electron dense cytoplasm containing numerous $\mathrm{L}$ and disrupted processes. $\mathrm{O}$ with electron dense cytoplasm containing numerous L. Activated Mg with numerous L. (C) Group IIb optic nerve shows A with large euchromatic nucleus and long processes containing bundles of $\mathrm{f}$ and L. O with dilated perinuclear cisternae (arrow head). A, astrocyte; f, filaments; L, lysosomes; Mf, myelinated optic nerve fibers; $\mathrm{Mg}$, microglia; $\mathrm{O}$, oligodendrocyte.

A

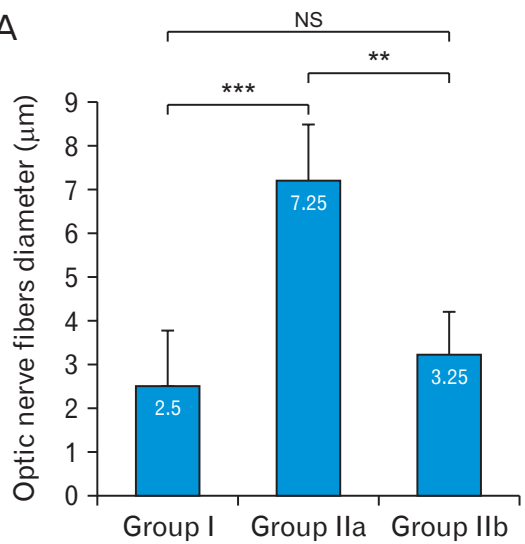

B

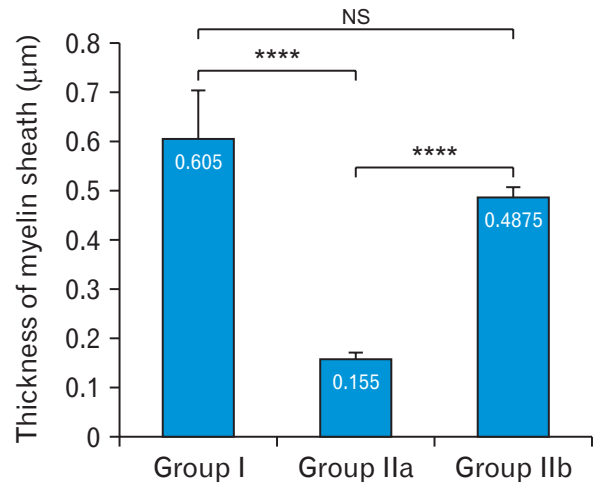

Fig. 7. Statistical analysis of the fiber's diameter and myelin thickness. (A) Comparison of the optic nerve fibers diameter ( $\mu \mathrm{m})$ in the studied groups. ${ }^{* * *} P<0.001 .{ }^{* *} P<0.01$. No significant difference, $P<0.3749$. (B) Comparison of the thickness of myelin sheath $(\mu \mathrm{m})$ of optic nerve fibers in the studied groups. NS, no significant. Data are expressed as mean \pm standard deviation. ${ }^{* * *} P<0.0001$. No significant difference, $P<0.05$. 
matic nucleus (Fig. 6A).

In group IIa, there was a reduction in the diameter of the optic nerve fibers compared to group I with thinning of myelin sheath. The mean diameter of optic nerve fibers was significantly decreased $(P$-value $<0.0002)$ compared to group I. It was $1.625 \pm 0.15$, ranged from 1.5 to 1.8 (Fig. $7 \mathrm{~A}$ ). The mean thickness of myelin sheath of optic nerve fibers was significantly decreased $(P$-value $<0.0001)$ compared to group I. It was $0.155 \pm 0.013$, ranged from 0.14 to 0.17 (Fig. $7 \mathrm{~B}$ ). Astrocytes were more electron-dense compared to group I with disrupted processes and their cytoplasm contained numerous lysosomes (Fig. 6B). Oligodendrocytes were more electron-dense compared to group I and their cytoplasm contained numerous lysosomes (Fig. 6B). Activated microglia with numerous lysosomes were observed (Fig. 6B).

In group IIb, there was an increase in the diameter of the optic nerve fibers compared to group IIa. The mean diameter of optic nerve fibers was significantly increased $(P$ value $<0.0012)$ compared to group IIa. It was $4.1575 \pm 0.8045$, ranged from 3.03 to 4.9 (Fig. 7A). The mean thickness of myelin sheath of optic nerve fibers was significantly increased $(P$-value $<0.0001)$ compared to group IIa. It was $0.4875 \pm 0.021$, ranged from 0.46 to 0.51 (Fig. 7B). Astrocytes were less electron-dense compared to group IIa. They had large nucleus with thin peripheral heterochromatin, long processes containing bundles of filaments and lysosomes (Fig. 6C). Oligodendrocytes were electron dense with dilated perinuclear cisternae (Fig. 6C). Less activated microglia with small oval heterochromatic nucleus and occasional lysosomes (Fig. 6C).

\section{Discussion}

In this work, we evaluated the protective effect of metformin on the rat optic nerve following photoreceptors loss induced by ENU. Photoreceptor loss initiates retinal remodeling, which ends in RGC death, the neurons that transmit the information to the brain via the optic nerve [1]. It was reported that ENU induced apoptosis of the photoreceptors in rodents 7 days after a single intraperitoneal injection via the downregulation of Bcl-2, upregulation of Bax and activation of the caspase families $[7,12]$. The retinal degenerative changes following photoreceptors loss induced by ENU will be discussed in another paper (in progress).

Group IIa (ENU-induced photoreceptor degeneration) of this study revealed a reduction in the diameter of the optic nerve fibers with thinning of myelin sheath confirmed by morphometric analysis. In agreement, axonal and myelin damage in optic nerve was detected in patients with inherited photoreceptor loss [13-15] Also, Similar findings were reported in transgenic rabbit model of inherited photoreceptor loss (RP) by Lahav et al. [16] and Asakawa et al. [17]. These authors hypothesized that the degenerative changes of optic nerve occurred as consequence to outer retinal degeneration.

On the other hand, statistical analysis of group IIb (metformin treated group) of this study revealed preservation of the diameter of optic nerve fibers and thickness of myelin sheath. This could correlate to the decreased oxidative stress and apoptosis detected by immunohistochemical study of iNOS and caspase-3 expression. In agreement, Oda [6] reported that metformin could protect against central and peripheral neuropathy in rat by decreasing oxidative stress and apoptosis via activation of adenosine monophosphateactivated protein kinase (AMPK) pathway. Also, Xu et al. [18] demonstrated that metformin could protect the optic nerve fibers by upregulation of crystallin proteins that enhance the survival of RGCs in transgenic rd1 mice (a model of photoreceptor degeneration).

The changes observed in optic nerve fibers in group IIa of this study were concomitant with morphological changes in oligodendrocytes suggested an impaired oligodendrocyte function. The progressive oligodendrocyte stress is followed by demyelination of the optic nerve [19, 20]. It was suggested that myelin repair could be performed by metformin via upregulation of factors that promote oligodendrocyte precursor cells differentiation and maturation, recruitment of oligodendrocyte precursor cells to the lesion site and induction of their maturation and down-regulation of myelin-associated neurite outgrowth inhibitor marker [21]. Also, Metformin attenuated oxidative stress with corresponding induction of antioxidative defenses in oligodendrocytes exposed to cytokines via AMPK activation [5, 22, 23]. Moreover, Kim et al. [24] reported that $\alpha \mathrm{B}$-crystallin protein was expressed in peripapillary glial cells and some oligodendrocytes. The crystallin proteins which might be upregulated by metformin were proved to be protective in nerve injury [25].

Activated microglial cells, characterized by small heterochromatic nucleus and scanty cytoplasm containing numerous lysosomes, were observed in group IIa. In addition, the number of CD68 positive cells was markedly increased in this group. It is known that CD68 marker is associated with phagocytic microglia [26]. In agreement, Gupta et al. [27] suggested that microglia are activated in response to pho- 
toreceptor death. In addition, immunohistochemical and morphometric results of this study revealed increased iNOS expression in the optic nerve of group IIa. It is known that phagocytic microglia (also known as M1 microglia) release proinflammatory cytokines such as tumour necrosis factor alpha (TNF- $\alpha$ ) and nitric oxide synthase-2 (NOS-2). TNF$\alpha$ binds to cell membrane and triggers apoptotic pathways while NOS-2 induces oxidative stress leading to caspase activation and mitochondrial dysfunction resulting in cell death $[28,29]$.

On the other hand, less activated microglial cells were observed in metformin-treated group of this study (group IIb). Statistically, the number of CD68 positive cells was significantly decreased compared to group IIa. There was an evidence that metformin inhibits microglia reactivity through different mechanisms including AMPK signaling, thereby reducing typical features of pro-inflammatory microglia including NADPH-dependent production of free radicals leading to polarization of microglia from proinflammatory phenotype (M1) to neuroprotective phenotype (M2) [30, 31].

Statistically, the number of astrocytes was decreased in group IIa optic nerve. Moreover, astrocytic processes were negatively stained with PTAH. The increased iNOS expression observed in this group indicated oxidative stress which might involve in the death of astrocytes [32]. Astrocytic clasmatodendrosis (lose their processes) was also observed in a previous study by Eltony and Abdelhameed [33] in optic nerve of Viagra treated rats. It was suggested that astrocytic clasmatodendrosis is related to excessive acidosis concomitant with hyperglycemia [34]. The hyperglycemia induced by ENU is thought to result from mutations in genes involved in glucose homeostasis $[35,36]$.

It is known that the optic nerve is subdivided into fascicles by connective tissue and glial septa [37]. The less organized septa observed in group IIa of this study was reported also by Asakawa et al. [17] in rabbit with photoreceptor degeneration. They attributed this disorganization to the enlargement of interstitial spaces due to the degeneration of myelinated axons.

Ultrastructurally, astrocytes of group IIa contained numerous lysosomes and lipofuscin granules. It has been reported that astrocytes participate in phagocytosis of cerebral white matter myelin in adult rabbit [38], in Wallerian degeneration of the opossum's optic nerve [39] and following brain trauma [40].

On the other hand, this study revealed that there was no statistically significant difference in the mean number of PTAH-stained astrocytes between metformin-treated group and the control group. Metformin was found to inhibit caspase-3 through activation of AMPK pathway. Thus, protect astrocytes from apoptosis induced by stress stimuli [41, 42].

In conclusion, metformin ameliorates the histological changes of the rat optic nerve following photoreceptors loss induced by ENU. This suggests the protective role of metformin in preventing optic nerve damage secondary to retinal photoreceptor loss.

\section{ORCID}

Sohair A. Eltony: https://orcid.org/0000-0003-2710-0565

Heba S. Mohaseb: https://orcid.org/0000-0002-8044-1812

Manal M. Sayed: https://orcid.org/0000-0003-0842-4548

Amel A. Ahmed: https://orcid.org/0000-0003-4388-1277

\section{Author Contributions}

Conceptualization: SAE, HSM, MMS. Data acquisition: SAE, HSM. Data analysis or interpretation: SAE, HSM, AAA. Drafting of the manuscript: SAE, MMS. Critical revision of the manuscript: MMS, SAE. Approval of the final version of the manuscript: all authors.

\section{Conflicts of Interest}

No potential conflict of interest relevant to this article was reported.

\section{Acknowledgements}

Research Funding Unit of Assiut University is the source of funding for this research.

\section{References}

1. García-Ayuso D, Di Pierdomenico J, Vidal-Sanz M, VillegasPérez MP. Retinal ganglion cell death as a late remodeling effect of photoreceptor degeneration. Int J Mol Sci 2019;20:4649.

2. Maaswinkel H, Mason B, Li L. ENU-induced late-onset night blindness associated with rod photoreceptor cell degeneration in zebrafish. Mech Ageing Dev 2003;124:1065-71.

3. Marco-Gomariz MA, Hurtado-Montalbán N, Vidal-Sanz M, Lund RD, Villegas-Pérez MP. Phototoxic-induced photoreceptor degeneration causes retinal ganglion cell degeneration in 
pigmented rats. J Comp Neurol 2006;498:163-79.

4. Garcia-Ayuso D, Di Pierdomenico J, Agudo-Barriuso M, VidalSanz M, Villegas-Pérez MP. Retinal remodeling following photoreceptor degeneration causes retinal ganglion cell death. Neural Regen Res 2018;13:1885-6.

5. Qi B, Hu L, Zhu L, Shang L, Sheng L, Wang X, Liu N, Wen N, Yu X, Wang Q, Yang Y. Metformin attenuates cognitive impairments in hypoxia-ischemia neonatal rats via improving remyelination. Cell Mol Neurobiol 2017;37:1269-78.

6. Oda SS. Metformin protects against experimental acrylamide neuropathy in rats. Drug Dev Res 2017;78:349-59.

7. Yoshizawa K, Sasaki T, Uehara N, Kuro M, Kimura A, Kinoshita Y, Miki H, Yuri T, Tsubura A. N-ethyl-N-nitrosourea induces retinal photoreceptor damage in adult rats. J Toxicol Pathol 2012;25:27-35.

8. Mao-Ying QL, Kavelaars A, Krukowski K, Huo XJ, Zhou W, Price TJ, Cleeland C, Heijnen CJ. The anti-diabetic drug metformin protects against chemotherapy-induced peripheral neuropathy in a mouse model. PLoS One 2014;9:e100701.

9. Zhao RR, Xu XC, Xu F, Zhang WL, Zhang WL, Liu LM, Wang WP. Metformin protects against seizures, learning and memory impairments and oxidative damage induced by pentylenetetrazole-induced kindling in mice. Biochem Biophys Res Commun 2014;448:414-7.

10. Drury RA, Wallington EA. Carleton's histological technique. 5th ed. Oxford: Oxford University Press; 1980. p. 15-21.

11. Gupta PD. Ultrastructural study on semithin section. Sci Tools 1983;30:6-7.

12. Yoshizawa K, Kuro-Kuwata M, Sasaki T, Lai YC, Kanematsu S, Miki H, Kimura-Kawanaka A, Uehara N, Yuri T, Tsubura A. Retinal degeneration induced in adult mice by a single intraperitoneal injection of N-ethyl-N-nitrosourea. Toxicol Pathol 2011;39:606-13.

13. Garcia-Martin E, Pinilla I, Sancho E, Almarcegui C, Dolz I, Rodriguez-Mena D, Fuertes I, Cuenca N. Optical coherence tomography in retinitis pigmentosa: reproducibility and capacity to detect macular and retinal nerve fiber layer thickness alterations. Retina 2012;32:1581-91.

14. Oishi A, Ogino K, Nakagawa S, Makiyama Y, Kurimoto M, Otani A, Yoshimura N. Longitudinal analysis of the peripapillary retinal nerve fiber layer thinning in patients with retinitis pigmentosa. Eye (Lond) 2013;27:597-604.

15. Zhang Q. Retinitis pigmentosa: progress and perspective. Asia Pac J Ophthalmol (Phila) 2016;5:265-71.

16. Lahav M, Craft J, Albert DM, Ishii Y. Advanced pigmentary retinal degeneration: an ultrastructural study. Retina 1982;2:6575.

17. Asakawa K, Ishikawa H, Uga S, Mashimo K, Kondo M, Terasaki $\mathrm{H}$. Histopathological changes of inner retina, optic disc, and optic nerve in rabbit with advanced retinitis pigmentosa. Neuroophthalmology 2016;40:286-91.

18. A L, Zou T, He J, Chen X, Sun D, Fan X, Xu H. Rescue of retinal degeneration in $\mathrm{rd} 1$ mice by intravitreally injected metformin. Front Mol Neurosci 2019;12:102.
19. Goldenberg-Cohen N, Guo Y, Margolis F, Cohen Y, Miller NR, Bernstein SL. Oligodendrocyte dysfunction after induction of experimental anterior optic nerve ischemia. Invest Ophthalmol Vis Sci 2005;46:2716-25.

20. Renner M, Stute G, Alzureiqi M, Reinhard J, Wiemann S, Schmid H, Faissner A, Dick HB, Joachim SC. Optic nerve degeneration after retinal ischemia/reperfusion in a rodent model. Front Cell Neurosci 2017;11:254.

21. Houshmand F, Barati M, Golab F, Ramezani-Sefidar S, Tanbakooie S, Tabatabaei M, Amiri M, Sanadgol N. Metformininduced AMPK activation stimulates remyelination through induction of neurotrophic factors, downregulation of NogoA and recruitment of Olig2+ precursor cells in the cuprizone murine model of multiple sclerosis. Daru 2019;27:583-92.

22. Paintlia A, Paintlia M, Mohan S, Singh A, Singh I. Antidiabetic drug, metformin protects oligodendrocytes under CNS pathological conditions: implication for multiple sclerosis (P5205). J Immunol 2013;190(1 Supplement):68.30.

23. Paintlia AS, Paintlia MK, Mohan S, Singh AK, Singh I. AMPactivated protein kinase signaling protects oligodendrocytes that restore central nervous system functions in an experimental autoimmune encephalomyelitis model. Am J Pathol 2013;183:526-41.

24. Kim JY, Sohn HJ, Seo JH. Characterization of the antigenic phenotype of $\alpha \mathrm{B}$-crystallin-expressing peripapillary glial cells in the developing chick retina. Anat Cell Biol 2011;44:35-40.

25. Wang YH, Li YC, Huo SJ, Yin ZQ. Alpha-crystallin promotes rat olfactory ensheathing cells survival and proliferation through regulation of PI3K/Akt/mTOR signaling pathways. Neurosci Lett 2012;531:170-5.

26. Zotova E, Bharambe V, Cheaveau M, Morgan W, Holmes C, Harris S, Neal JW, Love S, Nicoll JA, Boche D. Inflammatory components in human Alzheimer's disease and after active amyloid- $\beta 42$ immunization. Brain 2013;136(Pt 9):2677-96.

27. Gupta N, Brown KE, Milam AH. Activated microglia in human retinitis pigmentosa, late-onset retinal degeneration, and age-related macular degeneration. Exp Eye Res 2003;76:463-71.

28. Biswas SK, Mantovani A. Macrophage plasticity and interaction with lymphocyte subsets: cancer as a paradigm. Nat Immunol 2010;11:889-96.

29. Narayan DS, Wood JP, Chidlow G, Casson RJ. A review of the mechanisms of cone degeneration in retinitis pigmentosa. Acta Ophthalmol 2016;94:748-54.

30. Jin Q, Cheng J, Liu Y, Wu J, Wang X, Wei S, Zhou X, Qin Z, Jia J, Zhen X. Improvement of functional recovery by chronic metformin treatment is associated with enhanced alternative activation of microglia/macrophages and increased angiogenesis and neurogenesis following experimental stroke. Brain Behav Immun 2014;40:131-42.

31. Tayara K, Espinosa-Oliva AM, García-Domínguez I, Ismaiel AA, Boza-Serrano A, Deierborg T, Machado A, Herrera AJ, Venero JL, de Pablos RM. Divergent effects of metformin on an inflammatory model of Parkinson's disease. Front Cell Neurosci 2018;12:440. 
32. Means JC, Lopez AA, Koulen P. Resveratrol protects optic nerve head astrocytes from oxidative stress-induced cell death by preventing caspase- 3 activation, Tau dephosphorylation at Ser422 and formation of misfolded protein aggregates. Cell Mol Neurobiol 2020;40:911-26.

33. Eltony SA, Abdelhameed SY. Effect of chronic administration of sildenafil citrate (Viagra) on the histology of the retina and optic nerve of adult male rat. Tissue Cell 2017;49(2 Pt B):323-35.

34. Kraig RP, Chesler M. Astrocytic acidosis in hyperglycemic and complete ischemia. J Cereb Blood Flow Metab 1990;10:104-14.

35. Fenner D, Odili S, Hong HK, Kobayashi Y, Kohsaka A, Siepka SM, Vitaterna MH, Chen P, Zelent B, Grimsby J, Takahashi JS, Matschinsky FM, Bass J. Generation of N-ethyl-N-nitrosourea (ENU) diabetes models in mice demonstrates genotype-specific action of glucokinase activators. J Biol Chem 2011;286:3956072.

36. Bentley L, Esapa CT, Nesbit MA, Head RA, Evans H, Lath D, Scudamore CL, Hough TA, Podrini C, Hannan FM, Fraser WD, Croucher PI, Brown MA, Brown SD, Cox RD, Thakker RV. An N-ethyl-N-nitrosourea induced corticotropin-releasing hormone promoter mutation provides a mouse model for en- dogenous glucocorticoid excess. Endocrinology 2014;155:90822.

37. Sandell JH, Peters A. Effects of age on nerve fibers in the rhesus monkey optic nerve. J Comp Neurol 2001;429:541-53.

38. Vinores SA, Herman MM. Phagocytosis of myelin by astrocytes in explants of adult rabbit cerebral white matter maintained on Gelfoam matrix. J Neuroimmunol 1993;43:169-76.

39. Narciso MS, Hokoç JN, Martinez AM. Watery and dark axons in Wallerian degeneration of the opossum's optic nerve: different patterns of cytoskeletal breakdown? An Acad Bras Cienc 2001;73:231-43.

40. Jones RS, Minogue AM, Connor TJ, Lynch MA. Amyloid- $\beta$ induced astrocytic phagocytosis is mediated by CD36, CD47 and RAGE. J Neuroimmune Pharmacol 2013;8:301-11.

41. Blázquez C, Geelen MJ, Velasco G, Guzmán M. The AMPactivated protein kinase prevents ceramide synthesis de novo and apoptosis in astrocytes. FEBS Lett 2001;489:149-53.

42. Gabryel B, Liber S. Metformin limits apoptosis in primary rat cortical astrocytes subjected to oxygen and glucose deprivation. Folia Neuropathol 2018;56:328-36. 